Check for updates

Cite this: Phys. Chem. Chem. Phys., 2020, 22, 15770

\section{Correction: Assessing relative humidity dependent photoacoustics to retrieve mass accommodation coefficients of single optically trapped aerosol particles}

\author{
Matus E. Diveky, $\uparrow$ Sandra Roy, $\uparrow$ Johannes W. Cremer, Grégory David and \\ Ruth Signorell*
}

DOI: $10.1039 / \mathrm{d} 0 \mathrm{cp} 90148 \mathrm{~b}$

Correction for 'Assessing relative humidity dependent photoacoustics to retrieve mass accommodation coefficients of single optically trapped aerosol particles' by Matus E. Diveky et al., Phys. Chem. Chem. Phys., 2019, 21, 4721-4731, DOI: 10.1039/C8CP06980H.

rsc.li/pccp

(1) There is an error in the mass accommodation coefficient $\left(\alpha_{M}\right)$ values presented in the paper due to a typographical error in the $\beta_{\mathrm{i}}$ equations (eqn (3)) in the source code of the computer programs used. The trends discussed in the paper and the general results are not affected by this error. However, it increases the numerical values of $\alpha_{\mathrm{M}}$.

A simple correction formula can be applied to the reported values of $\alpha_{M}$ to account for this error:

$$
\alpha_{\mathrm{M}, \text { correct }}=\frac{1+\mathrm{Kn}_{\mathrm{M}}}{\alpha_{\mathrm{M}, \mathrm{old}}+\frac{\mathrm{K} n_{\mathrm{M}}}{\alpha_{\mathrm{M}, \mathrm{old}}}}
$$

Where $\alpha_{M, \text { old }}$ is the $\alpha_{M}$ reported in the paper, $\alpha_{\mathrm{M} \text {,correct }}$ represents correct values of $\alpha_{\mathrm{M}}$ and $\mathrm{Kn}_{\mathrm{M}}$ is the Knudsen number $\left(\lambda_{\mathrm{v}} / r\right)$. For $\alpha_{\mathrm{M}, \text { old }} \sim 1$ the correction can be neglected. Values of $\alpha_{\mathrm{M} \text {,correct }}>1$ (which can result for $\alpha_{\mathrm{M} \text {,old }} \sim 0.1$ ) are unphysical and are to be discarded. This does not influence the analysis as the corresponding simulations are virtually indistinguishable from those for $\alpha_{\mathrm{M}, \mathrm{old}}=1$.

A detailed list of all changes after applying the correction formula is provided as follows:

- In the abstract, "values of $a_{\mathrm{M}} \approx 0.02-0.005$ " should be modified to "values of $a_{\mathrm{M}} \approx 0.2-0.05$ "

- Page 4726 - The middle row of the original Fig. 4 corresponds to $\alpha_{\mathrm{M}, \text { correct }} \approx 0.02$ depending on $r$. In the figure caption, " $\alpha_{\mathrm{M}}=$ 0.001 " should be changed to " $\alpha_{M} \approx 0.02$ ". We verified that the simulations with a strictly fixed $\alpha_{M}=0.02$ produce nearly identical results to those shown in Fig. 4 (middle).

- Page 4727 - In the sentence "Fig. 4 (middle and bottom) shows the simulated PA signal as a function of particle radius for $\alpha_{M}$ values of 0.001 and 1.0." the values should be changed to " 0.02 and 1.0".

- Page 4728 - In Fig. 6, applying the correction to $\alpha_{\mathrm{M} \text {,old }}=0.1$ leads to $\alpha_{\mathrm{M} \text {,correct }}>1$, which is unphysical. The green line should therefore be discarded and the following sentences on p. 4728 should be removed: "Furthermore, the photoacoustic signals at $\alpha_{M}=0.1$ and $\alpha_{M}=1.0$ seem to overlap through all relative humidities for small particle sizes ( 0.9 and $2.1 \mu \mathrm{m}$ in Fig. 6), while for larger particles $(2.7 \mu \mathrm{m}$ in Fig. 6) these signals start to differ at higher relative humidities."

- Page 4728 - "For small particles, we find an accommodation coefficient value around $\sim 0.02$, while for larger particles the values decrease to less than 0.005 " should be modified to "For small particles, we find an accommodation coefficient value around $\sim 0.2$, while for larger particles the values decrease to less than 0.05 ".

- Page 4728 - In Fig. 7, the values for $\alpha_{\mathrm{M} \text {,correct }}$ now range from 0.2 to less than 0.05 .

- Page 4729 - "particles lies between $\sim 0.02$ and 0.005 at temperatures between 295-309 K" should be modified to "particles lies between $\sim 0.2$ and 0.05 at temperatures between $295-309 \mathrm{~K} "$

Laboratory of Physical Chemistry, Department of Chemistry and Applied Biosciences, ETH Zürich, Vladimir-Prelog-Weg 2, CH-8093 Zürich, Switzerland. E-mail: rsignorell@ethz.ch

$\dagger$ These authors contributed equally to this work. 
- In the Conclusions, "varied between 0.005-0.02 in the temperature range 295-309 K" should be modified to "varied between $0.2-0.05$ in the temperature range $295-309 \mathrm{~K}$ "

(2) Further notes:

- It should have been noted after eqn (9) that $f_{\mathrm{M}}$ was set to zero for the calculation of $\overline{T_{\mathrm{a}}}$ as there is no net mass flux at equilibrium.

- It should have been noted in eqn (6) and (9) that we assumed $\beta_{\mathrm{T}} \approx 1$.

- There is a typographical error in Table 2. $c_{\mathrm{p}, \mathrm{g}}$ should be replaced by $c_{\mathrm{p}, \mathrm{a}}$.

The Royal Society of Chemistry apologises for these errors and any consequent inconvenience to authors and readers. 